\title{
Kinetics-Limited Two-Step Growth of van der Waals Puckered Honeycomb Sb Monolayer
}

Zhi-Qiang Shi ${ }^{1 \dagger}$, Huiping Li ${ }^{2,3 \dagger}$, Qian-Qian Yuan ${ }^{1}$, Cheng-Long Xue ${ }^{1}$, Yong-Jie $\mathrm{Xu}^{1}$, Yang-Yang Lv ${ }^{4}$, Zhen-Yu Jia ${ }^{1}$, Yanbin Chen ${ }^{1,5}$, Wenguang $\mathrm{Zhu}^{2,3 *}$, ShaoChun $\mathrm{Li}^{1,5,6 *}$

1 National Laboratory of Solid State Microstructures, School of Physics, Nanjing University, Nanjing 210093, China

2 International Center for Quantum Design of Functional Materials (ICQD), Hefei National Laboratory for Physical Sciences at the Microscale, and Synergetic Innovation Center of Quantum Information and Quantum Physics, University of Science and Technology of China, Hefei, Anhui 230026, China

3 Key Laboratory of Strongly-Coupled Quantum Matter Physics Chinese Academy of Sciences, School of Physical Sciences, University of Science and Technology of China, Hefei, Anhui

$$
\text { 230026, China }
$$

4 National Laboratory of Solid State Microstructures, Department of Materials Science and Engineering, Nanjing University, Nanjing 210093, China

5 Collaborative Innovation Center of Advanced Microstructures, Nanjing University, Nanjing 210093, China

6 Jiangsu Provincial Key Laboratory for Nanotechnology, Nanjing University, Nanjing 210093, China.

$\uparrow$ These authors contributed equally to this work.

* Corresponding Authors: scli@nju.edu.cn; wgzhu@ustc.edu.cn. 
ABSTRACT: Puckered honeycomb Sb monolayer, the structural analog of black phosphorene, has been recently successfully grown by means of molecular beam epitaxy. However, little is known to date about the growth mechanism for such puckered honeycomb monolayer. In this study, by using scanning tunneling microscopy in combination with firstprinciples density functional theory calculations, we unveil that the puckered honeycomb Sb monolayer takes a kinetics-limited two-step growth mode. As the coverage of Sb increases, the Sb atoms firstly form the distorted hexagonal lattice as the half layer, and then the distorted hexagonal half-layer transforms into the puckered honeycomb lattice as the full layer. These results provide the atomic-scale insight in understanding the growth mechanism of puckered honeycomb monolayer, and can be instructive to the direct growth of other monolayers with the same structure.

KEYWORDS: 2D materials, monolayer antimonene, puckered honeycomb structure, molecular beam epitaxy, scanning tunneling microscopy, density functional theory calculations 
The puckered honeycomb lattice emerges as a particular member in the family of two dimensional or layered materials. The well-known example that hosts the puckered honeycomb structure is black phosphorene, which has found extensive interests since its rediscovery in 2014, owing to its exotic properties, such as high carrier mobility, tunable band gap, and anisotropic transport properties, etc. ${ }^{1-14}$ The bulk black phosphorous can be fabricated through either high pressure or catalyst assistance. ${ }^{15,16}$ However, the single layer of black phosphorous, namely black phosphorene, is only achievable via top-down methods of mechanical or chemical exfoliation. ${ }^{15,16}$ To directly grow the black phosphorene is rather difficult, even though the growth of its allotrope, blue phosphorene, has been experimentally and theoretically studied. ${ }^{17-19}$ On the other hand, the chemical instability of black phosphorene hinders the further exploration toward the application. An alternative effort is to search for the structural analog of black phosphorene in group V elements, e.g., As, $\mathrm{Sb}$ and $\mathrm{Bi}^{20-27}$

The bulk antimony crystallizes in a rhombohedral structure (Space group: $R \overline{3} m$ ) which is different from the black phosphorous. The corresponding single layer, namely $\beta$-antimonene, is in the buckled hexagonal lattice and has been well studied. ${ }^{28-38}$ Until recently, the large-scale and high-quality puckered honeycomb $\mathrm{Sb}$ monolayer, namely $\alpha$-antimonene, has been successfully grown on $T_{d}-\mathrm{WTe} 2$ substrate by using molecular beam epitaxy (MBE) technique. ${ }^{39}$ Further characterization indicates that the $\alpha-\mathrm{Sb} / \mathrm{WTe} \mathrm{H}_{2}$ is very stable upon exposure to air, and exhibits the linear electron band near Fermi energy. ${ }^{39}$ To date, a comprehensive understanding for the growth of such puckered honeycomb monolayer is still missing, which should be vital for driving the discovery of more practically useful monolayers with analogous structure.

In this study, we took the MBE-grown single layer $\alpha$-antimonene as an example, to explore its growth mechanism. Through tuning down the growth kinetics, we were able to 
characterize an intermediate metastable structure for the growing $\alpha$-antimonene monolayer by using scanning tunneling microscopy (STM). In combination with first-principles density functional theory (DFT) calculations, the growth mechanism was revealed. The growth of $\alpha$ antimonene can be separated into two stages. Initially, the adsorbed $\mathrm{Sb}$ atoms prefer to form a distorted hexagonal $(\mathrm{dH})$-like lattice, which acts as the half-layer transient state. The transient $\mathrm{dH}$ state is energetically metastable and eventually transforms into the puckered honeycomb $\alpha$ antimonene.

\section{RESULTS AND DISCUSSION:}

The puckered honeycomb lattice of $\alpha$-antimonene is composed of two atomic sublayers in vertical corrugation, as depicted in Figure 1a. There are four Sb atoms in each unit cell. Each $\mathrm{Sb}$ atom forms three covalent bonds with three neighboring atoms. The freestanding lattice constants of $\alpha$-antimonene are $4.36 \AA$ and $4.74 \AA$ along zigzag and armchair direction, respectively. ${ }^{22}$ Layered semiconductor SnSe crystal (orthorhombic structure, space group: Pnma $)^{40}$ was adopted as the substrate. The pristine surface of the cleaved SnSe is composed of the micrometer-scale and atomically-flat terrace, with a semiconducting band gap of $\sim 0.85 \mathrm{eV}$ (see Supporting Information Figure S1). Figure 1b shows the surface morphology of the $\alpha$-Sb

monolayer grown on the SnSe substrate kept at $\sim 400 \mathrm{~K}$. The large-scale $\alpha$-Sb monolayer islands form at this growth condition. Atomically resolved STM image, the inset to Figure 1b, confirms the well-ordered $\alpha$-Sb structure without obvious defects. In addition, the moiré pattern can be identified in Figure 1b, which originates from the minimal lattice mismatch between $\mathrm{Sb}$ and SnSe. 
(a)

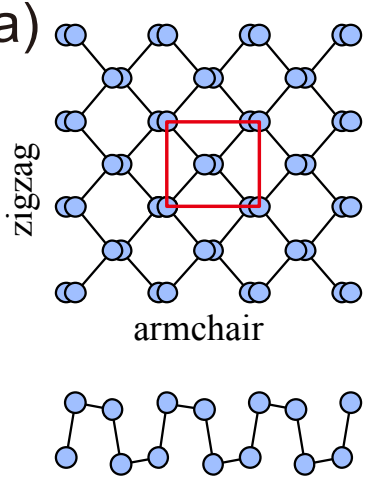

(b)

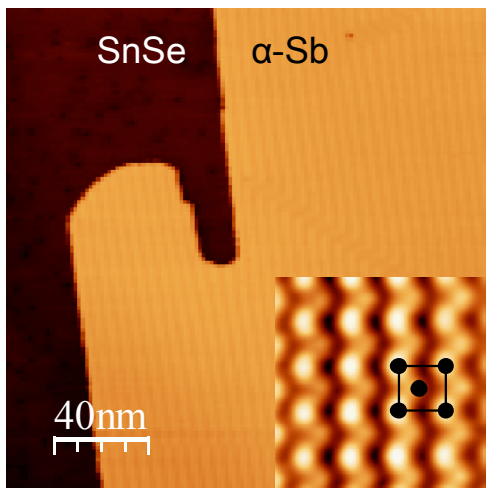

Figure 1. Epitaxial growth of single-layer $\alpha$-antimonene on the $\mathrm{SnSe}$ substrate. (a) The crystal structure of the freestanding $\alpha$-antimonene (upper: top view, lower: side view). The $1 \times 1$ unit cell is marked by the red rectangle. (b) The STM topographic image $\left(200 \times 200 \mathrm{~nm}^{2}\right)$ of the single-layer $\alpha$-antimonene epitaxially grown on the SnSe substrate. $U=+3 \mathrm{~V}, I_{\mathrm{t}}=100 \mathrm{pA}$. Inset: Atomically resolved STM image of the $\alpha$-antimonene. $U=-880 \mathrm{mV}, I_{\mathrm{t}}=5 \mathrm{nA}$. The $1 \times 1$ unit cell is marked by the black rectangle.

In order to investigate the growth mechanism in detail, an alternate growth recipe was adopted, i.e., to deposit $\mathrm{Sb}$ atoms onto the $\mathrm{SnSe}$ substrate kept at a lower temperature of $\sim 350 \mathrm{~K}$. In this way, the diffusion of the surface $\mathrm{Sb}$ atoms is limited and the phase transition from the intermediate state to $\alpha$-antimonene is effectively suppressed. Figure 2 a shows the morphology of $\mathrm{Sb}$ terrace grown at this condition. In contrast with the smooth Sb layer shown in Figure 1b, the surface becomes corrugated, which is mainly composed of two different regions, i.e., the protrusive and depressive regions. The protrusive region is structurally the same as the full monolayer of $\alpha$-antimonene. Line-scan profile measurement across the surface, as plotted in Figure $2 b$, indicates that the step height of protrusive island is $\sim 6.5 \AA$, consistent with the full monolayer of $\alpha-\mathrm{Sb}$. The depressive region is $\sim 1.6 \AA$ lower than the protrusive region, which is 
referred to as the half-layer $\mathrm{Sb}$ in the following. It is noteworthy that the measured height of the half-layer $\mathrm{Sb}, \sim 4.9 \AA$, is much larger than that of experimentally reported $\beta$-Sb monolayer. ${ }^{31}$ Post annealing of the surface without additional $\mathrm{Sb}$ deposition results in the increase of the areal proportion of the full monolayer $\alpha-\mathrm{Sb}$, at the expense of the half-layer $\mathrm{Sb}$, as demonstrated in Figure 2c,d. The half-layer regions finally disappear upon continuous annealing (see Supporting Information Figure S2). The STM results taken at various bias voltages in the same area confirm that the surface is stable and no scan-induced structural changes are observed (see Supporting Information Figure S3). It is thus indicated that the half-layer $\mathrm{Sb}$ is energetically metastable compared to the full monolayer $\alpha$-Sb, and undergoes a structural transition upon thermally annealing.

(a)

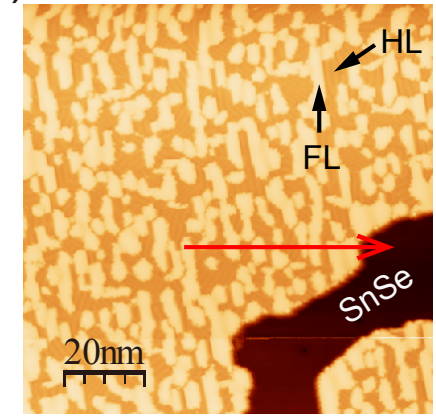

(c)

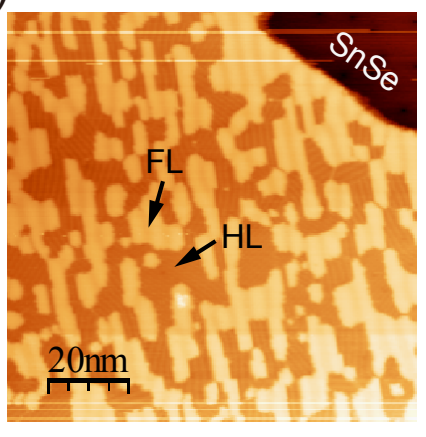

(b)

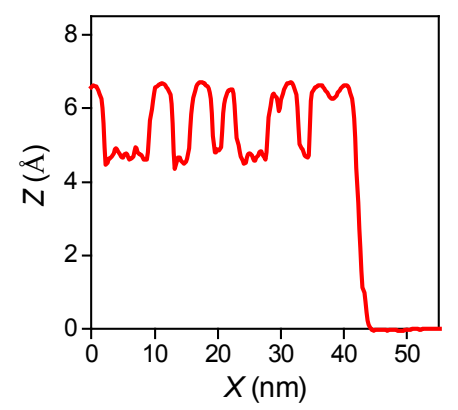

(d)

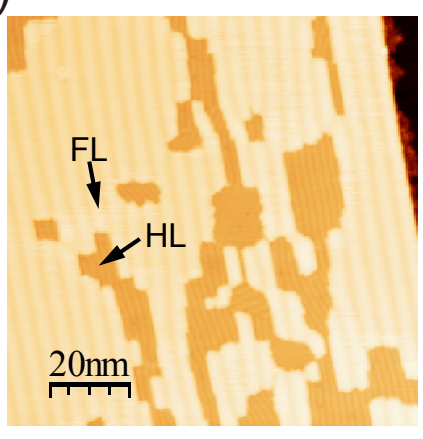

Figure 2. Morphology evolution of the $\mathrm{Sb}$ film deposited on the $\mathrm{SnSe}$ substrate upon post annealing. (a) The as-grown surface $\left(100 \times 100 \mathrm{~nm}^{2}\right)$ after $\sim 0.5 \mathrm{MLs}$ Sb are deposited on the SnSe substrate kept at $\sim 350 \mathrm{~K}$. $U=+3 \mathrm{~V}, I_{\mathrm{t}}=100 \mathrm{pA}$. (b) The 
line-scan profile taken along the red arrowed line in (a). The protrusive and depressed regions, as representatively marked by the arrows in ( $\mathrm{a}, \mathrm{c}, \mathrm{d})$, are assigned to the full layer (FL) of $\alpha$-antimonene and the half layer (HL) structure of Sb. The step height of the FL and HL Sb regions are $\sim 6.5 \AA$ and $\sim 4.9 \AA$, respectively. (c, d) The surface $\left(100 \times 100 \mathrm{~nm}^{2}\right)$ of the $\mathrm{Sb}$ film grown on SnSe after subsequently annealing at $\sim 350 \mathrm{~K}$ for $10 \mathrm{~min}$ (c) and $30 \mathrm{~min}$ (d), respectively. $U=+3 \mathrm{~V}, I_{\mathrm{t}}=100$ pA.

In the puckered honeycomb lattice, both of the top and bottom atomic layers host the same in-plane orthorhombic symmetry. Figure 3 shows the atomically resolved STM images taken on various half-layer Sb regions. The surface atoms are partially highlighted by yellow dots for better vision. Surprisingly, all the half-layer Sb regions exhibit the distorted hexagonal (dH) symmetry, which is completely different from the full monolayer $\alpha$-Sb. The moiré patterns of half-layer seem to indicate several different orientations, which can be ascribed to the different stacking order between the $\mathrm{dH}-\mathrm{Sb}$ and SnSe substrate. Meanwhile, the orientation of the full-layer $\alpha-\mathrm{Sb}$ is quite uniform on the substrate. Further structural analysis of the boundary, as shown in Supporting Information Figure S4, indicates that the atomic periodicities of the dHand $\alpha$-Sb monolayers are commensurately compatible along the direction of boundary, suggesting that the growth is likely to be seamless under the strain. In addition, there exists slight structural fluctuations among these half-layer regions, which further confirms their metastable nature. 
(a)

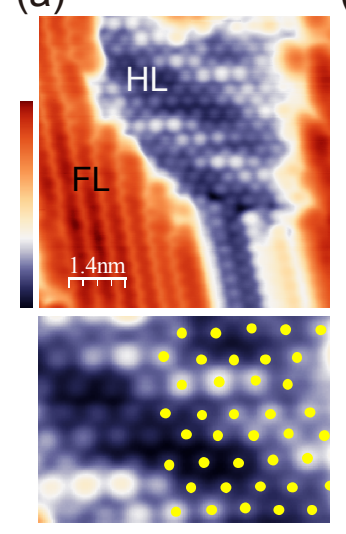

(b)

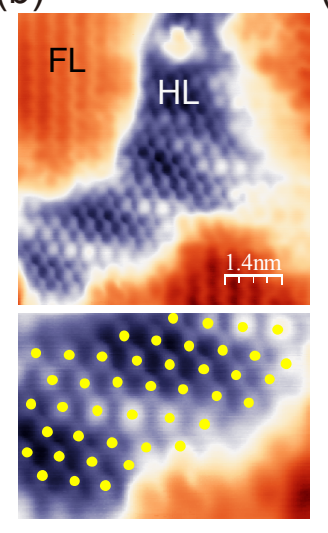

(c)

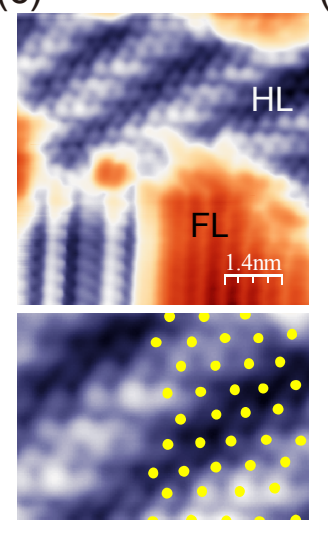

(d)

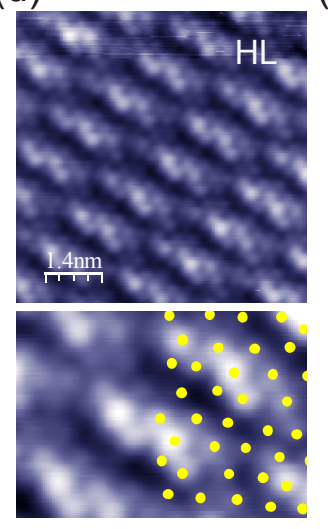

(e)

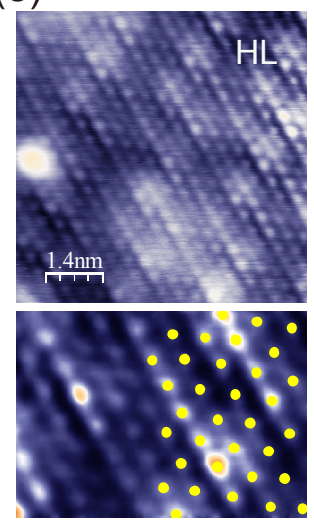

Figure 3. Various atomic structures of the half-layer Sb. (a-c) Atomically resolved images taken on the as-grown sample. (d, e) Atomically resolved images taken on the sample after thermally annealing at $350 \mathrm{~K}$ for $30 \mathrm{~min}$. Upper panel: large-scale images $\left(7 \times 7 \mathrm{~nm}^{2}\right) . U=-1.3 \mathrm{~V} ; I_{\mathrm{t}}=1 \mathrm{nA}$. Lower panel: Zoom-in images $(3.5 \times 2.5$ $\mathrm{nm}^{2}$ ) extracted from the upper panel. The regions of the half-layer (HL) Sb and full layer (FL) $\alpha$-Sb are labeled on the images. The yellow dot arrays are partially superimposed on the surface atoms for better vision.

Based on DFT calculations, we identified the $\mathrm{dH}$ structure as shown in Supporting Information Figure S5. The calculated density of state of this half-layer dH structure shows a prominent band gap of $0.6 \mathrm{eV}$. Considering that DFT calculated band gaps at GGA level are normally severely underestimated by roughly half, the calculated band gap is comparable with the STS measured value of $1.0 \mathrm{eV}$ taken on the half-layer region, as displayed in Supporting Information Figure S6. The formation energy calculations also confirm that the $\mathrm{dH}$ structure is less stable than the puckered $\alpha$-Sb monolayer on the SnSe substrate with a formation energy difference of $59 \mathrm{meV} / \mathrm{atom}$. Regarding to the different orientation of morié patterns observed in the half-layer $\mathrm{dH}-\mathrm{Sb}$, we performed DFT calculations to evaluate the binding energy of $\mathrm{dH}-\mathrm{Sb}$ and $\alpha-\mathrm{Sb}$ on $\mathrm{SnSe}$ substrate with different orientations. The results, as shown in Supporting 
Information Figure $\mathrm{S} 7$, indicate that the $\mathrm{dH}-\mathrm{Sb}$ is stable with very similar binding energy in several different orientations. However, the $\alpha$-Sb dominantly favors the specific orientation and thus is aligned with the SnSe substrate.

We further explored possible kinetic pathways of the structural transformation from the metastable half-layer dH structure to the ground-state full-layer puckered structure. For simplicity, free-standing monolayers were used in the calculations. We first considered the traditional direct process shown in Figure 4, a kinetic pathway similar to that for the transformation from a buckled honeycomb blue $\mathrm{P}$ to a puckered black $\mathrm{P} .{ }^{41}$ During the phase transformation from $\mathrm{dH}-\mathrm{Sb}$ to $\alpha$-Sb, as illustrated in Supporting Information Figure S8(a), one of every two rows of atoms along the zigzag direction in the upper layer flips to the lower layer, accompanied by one row of the adjacent atoms in the lower layer flipping inversely to the upper layer, without bond breaking. The calculated activation barrier of the process is $525 \mathrm{meV} /$ atom. However, a more feasible kinetic process with a lower activation barrier is revealed via a twostep motion as illustrated in Figure 4 and Supporting Information Figure S8(b). In the first step, the $\mathrm{dH}$ structure transforms into a metastable intermediate structure through an in-plane compression along the armchair direction with an activation barrier of $203 \mathrm{meV} /$ atom. Then the structure converts into the puckered structure through a bonding rearrangement process between the $\mathrm{Sb}$ atoms by overcoming an activation barrier of $145 \mathrm{meV} / \mathrm{atom}$. The overall activation barrier of this two-step process is $241 \mathrm{meV} / \mathrm{atom}$, which is expected to be accessible at the experimental temperatures. In addition, the kinetics of a nucleation-propagation process was studied by using a rectangular stripe supercell, as illustrated in Supporting Information Figure S9. A unit of the $\alpha$ phase first nucleates in a dH-Sb background by overcoming a barrier of 144 $\mathrm{meV} / \mathrm{atom}$, and propagates over the whole supercell subsequently through even smaller barriers 
of around $110 \mathrm{meV} /$ atom. These results indicate the transition from $\mathrm{dH}$ to $\alpha$ phase is feasible and more likely to occur experimentally. Unfortunately, the intermediate state is not experimentally observed. According to our DFT calculation, the energy barriers required to overcome from the intermediate state to the $\alpha-\mathrm{Sb}$ or $\mathrm{dH}-\mathrm{Sb}$ are $\sim 145 \mathrm{meV} /$ atom, which is so small that the intermediate state can readily transform into the $\alpha$ - or $\mathrm{dH}$ state upon annealing at $350 \mathrm{~K}$.

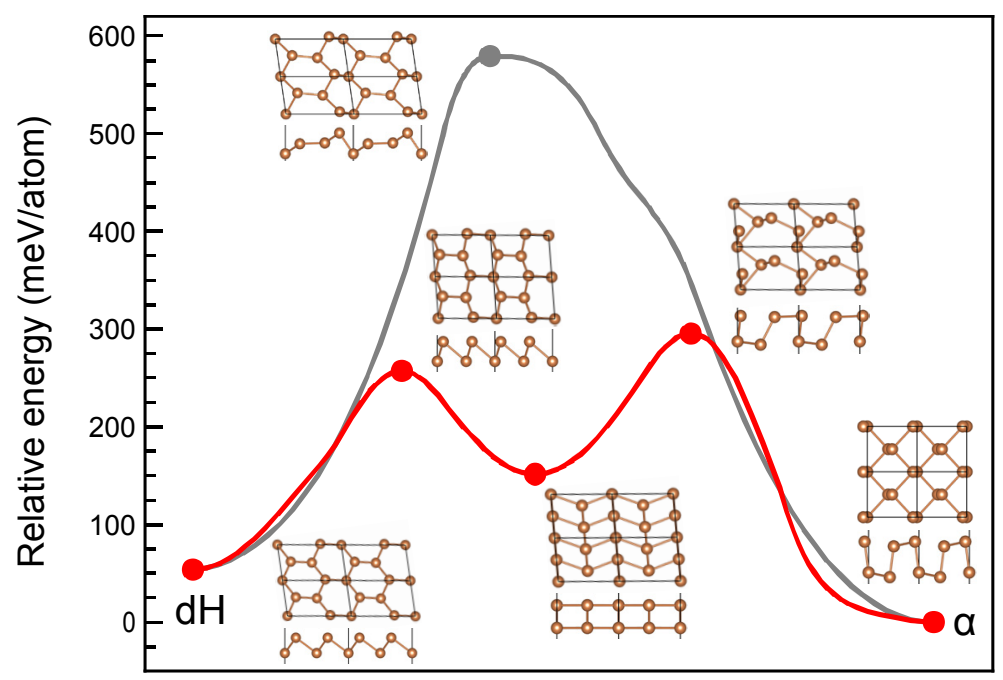

Reaction coordinate

Figure 4. Kinetic pathways from the $\mathrm{dH}$ structure to the puckered $\alpha$-Sb layer through a traditional direct process (grey path) and a two-step process (red path). Insets show the atomic structures of the initial $\mathrm{dH}$ structure, the intermediate structure, the final $\alpha-\mathrm{Sb}$ structure, and the transition states. The points marked on the energy profile indicate the position of each corresponding atomic structure.

To exclude the impact of substrate on the formation of the hexagonal-like half-layer $\mathrm{Sb}$, we explored a different substrate of $T_{d}-\mathrm{MoTe}_{2}$ (Space group: $\left.P m n{ }_{2}\right) .{ }^{42}$ The STM images taken on $\mathrm{Sb} / \mathrm{MoTe}_{2}$ are shown in Supporting Information Figure S10, where the monolayer (1L) and bilayer (2L) $\alpha$-antimonene, as well as the intermediate half-layer (1.5L) region, can be identified. 
Consistent with the $\mathrm{Sb}$ on $\mathrm{SnSe}$, the half-layer $\mathrm{Sb}$ formed on the $T_{d}-\mathrm{MoTe}_{2}$ substrate also exhibits the hexagonal-like lattice symmetry. Moreover, such half-layer Sb can also form on top of the $\alpha$ $\mathrm{Sb}$ monolayer instead of the $\mathrm{MoTe}_{2}$ substrate. The similar phenomenon is also observed when directly depositing Sb over the $\alpha$-Sb monolayer on $\mathrm{SnSe}$ (see Supporting Information Figure S11). Therefore, the influence of substrate to the half-layer formation can be ruled out.

A recent literature reported the substrate mediated phase transition from $\alpha$-Sb to $\beta$-Sb monolayer on $\mathrm{Bi}_{2} \mathrm{Se}_{3}$ substrate. ${ }^{43}$ In contrast, here we demonstrate the intrinsic growth kinetics of $\alpha$-Sb without the influence of substrate.

\section{CONCLUSIONS:}

In summary, the growth mechanism of the $\alpha$-antimonene monolayer has been investigated. Combining the experiment and DFT calculations, a two-step growth mode is unveiled. A distorted hexagonal atomic layer as the half-layer meta-structure is formed, prior to the transformation to the full puckered honeycomb monolayer. We believe such a two-step growth behavior should be universal to other monolayers in analogous structure, regardless of the choice of substrate. 


\section{METHODS:}

The $\alpha$-antimonene was grown and characterized in a commercial STM-MBE combined system (Unisoku USM-1500). The base pressure is $1 \times 10^{-10}$ Torr. The SnSe and $T_{\mathrm{d}}-\mathrm{MoTe}_{2}$ substrates were cleaved under ultrahigh vacuum, and the surface morphologies were verified in situ by STM prior to the sample growth. High purity (99.999\%) Sb was loaded in a standard Knudsen evaporator. The flux was kept at $\sim 0.3$ monolayers (MLs) per minute. All the STM measurements were performed at $78 \mathrm{~K}$ with a mechanically polished PtIr tip. The sample was taken out of the STM stage and transferred to the preparation chamber prior to the post annealing process.

Density functional calculations were performed by using Vienna ab Initio simulation package (VASP). ${ }^{44}$ The projector-augmented wave pseudopotentials with the Perdew-BurkeErnzerhof type of generalized gradient approximation (GGA-PBE) were used. ${ }^{45}$ The energy cutoff of the plane wave basis was set to $300 \mathrm{eV}$ and a vacuum of more than $15 \AA$ was introduced to the slabs. Van der Waals corrections of DFT-D2 ${ }^{46}$ method were treated in all vdW heterostructure systems. Optimized atomic structures were achieved until the forces on each atom were smaller than $0.01 \mathrm{eV} / \AA$. The climbing-image nudged elastic band $(\mathrm{cNEB})^{47} \mathrm{method}$ was used to simulate the phase transition process. 


\section{AUTHOR CONTRIBUTIONS}

S.-C.L. conceived the project. Z.-Q.S., Q.-Q.Y., and C.-L.X. prepared the samples and carried out STM experiments with the assistance of Y.-J.X. and Z.-Y.J. Y.-Y.L. and Y.-B.C. grew the substrate single crystals. H.L. and W.Z. carried out theoretical calculations. Z.-Q.S., H.L., W.Z., and S.-C.L. prepared the manuscript. All authors discussed the results and commented on the manuscript.

\section{ACKNOWLEDGMENT}

This work was financially supported by the National Natural Science Foundation of China (Grants Nos. 11774149, 11790311, 11674299, and 11634011, 51872134, 11574131, 51902152), the Foundation for Innovative Research group of the National Natural Science Foundation of China (No. 51721001); the National Key Research and Development Program of China (Grant Nos. 2017YFA0204904 and 2019YFA0210004), the Strategic Priority Research Program of Chinese Academy of Sciences (Grant No. XDB30000000), Anhui Initiative in Quantum Information Technologies (Grant No. AHY170000), and the Fundamental Research Funds for the Central Universities (Grant Nos. WK2340000082 and WK2060190084). 


\section{REFERENCES}

(1) Li, L.; Yu, Y.; Ye, G. J.; Ge, Q.; Ou, X.; Wu, H.; Feng, D.; Chen, X. H.; Zhang, Y. Black Phosphorus Field-Effect Transistors. Nat. Nanotechnol. 2014, 9, 372.

(2) Liu, H.; Neal, A. T.; Zhu, Z.; Luo, Z.; Xu, X.; Tománek, D.; Ye, P. D. Phosphorene: An Unexplored 2D Semiconductor with a High Hole Mobility. ACS Nano 2014, 8, 4033.

(3) Qiao, J.; Kong, X.; Hu, Z.-X.; Yang, F.; Ji, W. High-Mobility Transport Anisotropy and Linear Dichroism in Few-Layer Black Phosphorus. Nat. Commun. 2014, 5, 4475.

(4) Xia, F.; Wang, H.; Jia, Y. Rediscovering Black Phosphorus as an Anisotropic Layered Material for Optoelectronics and Electronics. Nat. Commun. 2014, 5, 4458.

(5) Tran, V.; Soklaski, R.; Liang, Y.; Yang, L. Layer-Controlled Band Gap and Anisotropic Excitons in Few-Layer Black Phosphorus. Phys. Rev. B 2014, 89, 235319.

(6) Rodin, A. S.; Carvalho, A.; Castro Neto, A. H. Strain-Induced Gap Modification in Black Phosphorus. Phys. Rev. Lett. 2014, 112, 176801.

(7) Favron, A.; Gaufrès, E.; Fossard, F.; Phaneuf-L'Heureux, A.-L.; Tang, N. Y.-W.; Lévesque, P. L.; Loiseau, A.; Leonelli, R.; Francoeur, S.; Martel, R. Photooxidation and Quantum Confinement Effects in Exfoliated Black Phosphorus. Nat. Mater. 2015, 14, 826.

(8) Xiang, Z. J.; Ye, G. J.; Shang, C.; Lei, B.; Wang, N. Z.; Yang, K. S.; Liu, D. Y.; Meng, F. B.; Luo, X. G.; Zou, L. J.; Sun, Z.; Zhang, Y.; Chen, X. H. Pressure-Induced Electronic Transition in Black Phosphorus. Phys. Rev. Lett. 2015, 115, 186403.

(9) Kim, J.; Baik, S. S.; Ryu, S. H.; Sohn, Y.; Park, S.; Park, B.-G.; Denlinger, J.; Yi, Y.; Choi, H. J.; Kim, K. S. Observation of Tunable Band Gap and Anisotropic Dirac Semimetal State in Black Phosphorus. Science 2015, 349, 723.

(10) Wang, X.; Jones, A. M.; Seyler, K. L.; Tran, V.; Jia, Y.; Zhao, H.; Wang, H.; Yang, L.; Xu, X.; Xia, F. Highly Anisotropic and Robust Excitons in Monolayer Black Phosphorus. Nat. Nanotechnol. 2015, 10, 517.

(11) Li, L.; Ye, G. J.; Tran, V.; Fei, R.; Chen, G.; Wang, H.; Wang, J.; Watanabe, K.; Taniguchi, T.; Yang, L.; Chen, X. H.; Zhang, Y. Quantum Oscillations in a Two-Dimensional Electron Gas in Black Phosphorus Thin Films. Nat. Nanotechnol. 2015, 10, 608.

(12) Li, L.; Yang, F.; Ye, G. J.; Zhang, Z.; Zhu, Z.; Lou, W.; Zhou, X.; Li, L.; Watanabe, K.; Taniguchi, T.; Chang, K.; Wang, Y.; Chen, X. H.; Zhang, Y. Quantum Hall Effect in Black Phosphorus Two-Dimensional Electron System. Nat. Nanotechnol. 2016, 11, 593.

(13) Liu, Y.; Rodrigues, J. N. B.; Luo, Y. Z.; Li, L.; Carvalho, A.; Yang, M.; Laksono, E.; Lu, J.; Bao, Y.; Xu, H.; Tan, S. J. R.; Qiu, Z.; Sow, C. H.; Feng, Y. P.; Castro Neto, A. H.; Adam, S.; Lu, J.; Loh, K. P. Tailoring Sample-Wide Pseudo-Magnetic Fields on a Graphene-Black Phosphorus Heterostructure. Nat. Nanotechnol. 2018, 13, 828.

(14) Gao, A.; Lai, J.; Wang, Y.; Zhu, Z.; Zeng, J.; Yu, G.; Wang, N.; Chen, W.; Cao, T.; Hu, W.; Sun, D.; Chen, X.; Miao, F.; Shi, Y.; Wang, X. Observation of Ballistic Avalanche Phenomena in Nanoscale Vertical InSe/BP Heterostructures. Nat. Nanotechnol. 2019, 14, 217.

(15) Liu, H.; Du, Y.; Deng, Y.; Peide, D. Y. Semiconducting Black Phosphorus: Synthesis, Transport Properties and Electronic Applications. Chem. Soc. Rev. 2015, 44, 2732.

(16) Carvalho, A.; Wang, M.; Zhu, X.; Rodin, A. S.; Su, H.; Castro Neto, A. H. Phosphorene: From Theory to Applications. Nat. Rev. Mater. 2016, 1, 16061.

(17) Zhang, J. L.; Zhao, S.; Han, C.; Wang, Z.; Zhong, S.; Sun, S.; Guo, R.; Zhou, X.; Gu, C. D.; Yuan, K. D.; Li, Z.; Chen, W. Epitaxial Growth of Single Layer Blue Phosphorus: A New Phase of Two-Dimensional Phosphorus. Nano Lett. 2016, 16, 4903. 
(18) Xu, J.-P.; Zhang, J.-Q.; Tian, H.; Xu, H.; Ho, W.; Xie, M. One-Dimensional Phosphorus Chain and Two-Dimensional Blue Phosphorene Grown on Au(111) by Molecular-Beam Epitaxy. Phys. Rev. Mater. 2017, 1, 061002.

(19) Zeng, J.; Cui, P.; Zhang, Z. Half Layer By Half Layer Growth of a Blue Phosphorene Monolayer on a GaN(001) Substrate. Phys. Rev. Lett. 2017, 118, 046101.

(20) Zhang, S.; Yan, Z.; Li, Y.; Chen, Z.; Zeng, H. Atomically Thin Arsenene and Antimonene: Semimetal-Semiconductor and Indirect-Direct Band-Gap Transitions. Angew. Chem., Int. Ed. 2015, 54, 3112.

(21) Aktürk, O. Ü.; Özçelik, V. O.; Ciraci, S. Single-Layer Crystalline Phases of Antimony: Antimonenes. Phys. Rev. B 2015, 91, 235446.

(22) Wang, G.; Pandey, R.; Karna, S. P. Atomically Thin Group V Elemental Films: Theoretical Investigations of Antimonene Allotropes. ACS Appl. Mater. Interfaces 2015, 7, 11490.

(23) Lu, Y.; Xu, W.; Zeng, M.; Yao, G.; Shen, L.; Yang, M.; Luo, Z.; Pan, F.; Wu, K.; Das, T.;

He, P.; Jiang, J.; Martin, J.; Feng, Y. P.; Lin, H.; Wang, X.-S. Topological Properties Determined by Atomic Buckling in Self-Assembled Ultrathin Bi(110). Nano Lett. 2015, 15, 80.

(24) Luo, K.; Chen, S.; Duan, C. Indirect-Direct Band Gap Transition of Two-Dimensional Arsenic Layered Semiconductors-Cousins of Black Phosphorus. Sci. China-Phys. Mech. Astron. 2015, 58, 087301.

(25) Kamal, C.; Ezawa, M. Arsenene: Two-Dimensional Buckled and Puckered Honeycomb Arsenic Systems. Phys. Rev. B 2015, 91, 085423.

(26) Lu, Y.; Zhou, D.; Chang, G.; Guan, S.; Chen, W.; Jiang, Y.; Jiang, J.; Wang, X.-S.; Yang, S. A.; Feng, Y. P.; Kawazoe, Y.; Lin, H. Multiple Unpinned Dirac Points in Group-Va SingleLayers with Phosphorene Structure. npj Comput. Mater. 2016, 2, 16011.

(27) Zhang, S.; Guo, S.; Chen, Z.; Wang, Y.; Gao, H.; Gómez-Herrero, J.; Ares, P.; Zamora, F.; Zhu, Z.; Zeng, H. Recent Progress in 2D Group-VA Semiconductors: From Theory to

Experiment. Chem. Soc. Rev. 2018, 47, 982.

(28) Zhang, P.; Liu, Z.; Duan, W.; Liu, F.; Wu, J. Topological and Electronic Transitions in A Sb(111) Nanofilm: The Interplay between Quantum Confinement and Surface Effect. Phys. Rev. $B$ 2012, 85, 201410.

(29) Bian, G.; Wang, X.; Liu, Y.; Miller, T.; Chiang, T.-C. Interfacial Protection of Topological Surface States in Ultrathin Sb Films. Phys. Rev. Lett. 2012, 108, 176401.

(30) Ji, J.; Song, X.; Liu, J.; Yan, Z.; Huo, C.; Zhang, S.; Su, M.; Liao, L.; Wang, W.; Ni, Z.; Hao, Y.; Zeng, H. Two-Dimensional Antimonene Single Crystals Grown by van der Waals Epitaxy. Nat. Commun. 2016, 7, 13352.

(31) Wu, X.; Shao, Y.; Liu, H.; Feng, Z.; Wang, Y.-L.; Sun, J.-T.; Liu, C.; Wang, J.-O.; Liu, Z.L.; Zhu, S.-Y.; Wang, Y.-Q.; Du, S.-X.; Shi, Y.-G.; Ibrahim, K.; Gao, H.-J. Epitaxial Growth and Air-Stability of Monolayer Antimonene on PdTe 2 . Adv. Mater. 2017, 29, 1605407.

(32) Li, G.; Hanke, W.; Hankiewicz, E. M.; Reis, F.; Schäfer, J.; Claessen, R.; Wu, C.; Thomale, R. Theoretical Paradigm for the Quantum Spin Hall Effect at High Temperatures. Phys. Rev. B 2018, 98, 165146.

(33) Fortin-Deschênes, M.; Waller, O.; Menteş, T. O.; Locatelli, A.; Mukherjee, S.; Genuzio, F.; Levesque, P. L.; Hébert, A.; Martel, R.; Moutanabbir, O. Synthesis of Antimonene on Germanium. Nano Lett. 2017, 17, 4970.

(34) Shao, Y.; Liu, Z.-L.; Cheng, C.; Wu, X.; Liu, H.; Liu, C.; Wang, J.-O.; Zhu, S.-Y.; Wang, Y.-Q.; Shi, D.-X.; Ibrahim, K.; Sun, J.-T.; Wang, Y.-L.; Gao, H.-J. Epitaxial Growth of Flat Antimonene Monolayer: A New Honeycomb Analogue of Graphene. Nano Lett. 2018, 18, 2133. 
(35) Mao, Y.-H.; Zhang, L.-F.; Wang, H.-L.; Shan, H.; Zhai, X.-F.; Hu, Z.-P.; Zhao, A.-D.; Wang, B. Epitaxial Growth of Highly Strained Antimonene on Ag(111). Front. Phys. 2018, 13, 138106.

(36) Zhu, S.-Y.; Shao, Y.; Wang, E.; Cao, L.; Li, X.-Y.; Liu, Z.-L.; Liu, C.; Liu, L.-W.; Wang, J.-O.; Ibrahim, K.; Sun, J.-T.; Wang, Y.-L.; Du, S.; Gao, H.-J. Evidence of Topological Edge States in Buckled Antimonene Monolayers. Nano Lett. 2019, 19, 6323.

(37) Niu, T.; Zhou, W.; Zhou, D.; Hu, X.; Zhang, S.; Zhang, K.; Zhou, M.; Fuchs, H.; Zeng, H. Modulating Epitaxial Atomic Structure of Antimonene through Interface Design. Adv. Mater.

2019, 31, 1902606.

(38) Niu, T.; Meng, Q.; Zhou, D.; Si, N.; Zhai, S.; Hao, X.; Zhou, M.; Fuchs, H. Large - Scale Synthesis of Strain - Tunable Semiconducting Antimonene on Copper Oxide. Adv. Mater. 2019, 32, 1906873.

(39) Shi, Z.-Q.; Li, H.; Yuan, Q.-Q.; Song, Y.-H.; Lv, Y.-Y.; Shi, W.; Jia, Z.-Y.; Gao, L.; Chen, Y.-B.; Zhu, W.; Li, S.-C. Van der Waals Heteroepitaxial Growth of Monolayer Sb in a Puckered Honeycomb Structure. Adv. Mater. 2019, 31, 1806130.

(40) Zhao, L.-D.; Lo, S.-H.; Zhang, Y.; Sun, H.; Tan, G.; Uher, C.; Wolverton, C.; Dravid, V. P.; Kanatzidis, M. G. Ultralow Thermal Conductivity and High Thermoelectric Figure of Merit in SnSe Crystals. Nature 2014, 508, 373.

(41) Zhu, Z.; Tománek, D. Semiconducting Layered Blue Phosphorus: A Computational Study. Phys. Rev. Lett. 2014, 112, 176802.

(42) Deng, K.; Wan, G.; Deng, P.; Zhang, K.; Ding, S.; Wang, E.; Yan, M.; Huang, H.; Zhang, H.; Xu, Z.; Denlinger, J.; Fedorov, A.; Yang, H.; Duan, W.; Yao, H.; Wu, Y.; Fan, S.; Zhang, H.; Chen, X.; Zhou, S. Experimental Observation of Topological Fermi Arcs in Type-II Weyl Semimetal MoTe. Nat. Phys. 2016, 12, 1105.

(43) Hogan, C.; Holtgrewe, K.; Ronci, F.; Colonna, S.; Sanna, S.; Moras, P.; Sheverdyaeva, P. M.; Mahatha, S.; Papagno, M.; Aliev, Z. S.; Babanly, M.; Chulkov, E. V.; Carbone, C.;

Flammini, R. Temperature Driven Phase Transition at the Antimonene/ $\mathrm{Bi}_{2} \mathrm{Se}_{3}$ van der Waals Heterostructure. Acs Nano 2019, 13, 10481.

(44) Kresse, G.; Furthmüller, J. Efficient Iterative Schemes for ab Initio Total-Energy Calculations Using a Plane-Wave Basis Set. Phys. Rev. B 1996, 54, 11169.

(45) Perdew, J. P.; Burke, K.; Ernzerhof, M. Generalized Gradient Approximation Made Simple. Phys. Rev. Lett. 1996, 77, 3865.

(46) Grimme, S. Semiempirical GGA-Type Density Functional Constructed with a Long-Range Dispersion Correction. J. Comput. Chem. 2006, 27, 1787.

(47) Henkelman, G.; Uberuaga, B. P.; Jónsson, H. A Climbing Image Nudged Elastic Band Method for Finding Saddle Points and Minimum Energy Paths. J. Chem. Phys. 2000, 113, 9901. 